\title{
Exercise Therapy for Fibromyalgia
}

\author{
Angela J. Busch • Sandra C. Webber • Mary Brachaniec • Julia Bidonde • \\ Vanina Dal Bello-Haas • Adrienne D. Danyliw • Tom J. Overend • Rachel S. Richards • \\ Anuradha Sawant • Candice L. Schachter
}

Published online: 5 July 2011

(C) The Author(s) 2011. This article is published with open access at Springerlink.com

\begin{abstract}
Fibromyalgia syndrome, a chronic condition typically characterized by widespread pain, nonrestorative sleep, fatigue, cognitive dysfunction, and other somatic symptoms, negatively impacts physical and emotional function and reduces quality of life. Exercise is commonly recommended in the management of people with fibromyalgia, and interest in examining exercise benefits for those with the syndrome has grown substantially over the past 25 years. Research supports aerobic and strength training to improve physical fitness and function, reduce fibromyalgia symptoms, and improve quality of life. However, other forms of exercise (e.g., tai chi, yoga, Nordic walking, vibration techniques) and lifestyle physical activity also have been investigated to determine their effects. This paper highlights findings from recent randomized controlled trials and reviews of exercise for people with fibromyalgia, and includes information regarding factors that influence response and adherence to exercise to assist clinicians with exercise and physical activity prescription decision-making to optimize health and well-being.
\end{abstract}

A. J. Busch $(\bowtie) \cdot$ S. C. Webber • V. D. Bello-Haas •

C. L. Schachter

School of Physical Therapy, University of Saskatchewan,

1121 College Drive,

Saskatoon, Saskatchewan S7N 0W3, Canada

e-mail: angela.busch@usask.ca

\section{Brachaniec}

Cochrane Musculoskeletal Group \& CIHR-IMHA Knowledge

Exchange Task Force,

229 Bessborough Avenue,

Moncton, New Brunswick E1E 1R3, Canada

J. Bidonde

Community Health \& Epidemiology, University of Saskatchewan,

501-121 Research Drive,

Saskatoon, Saskatchewan S7N 1K2, Canada
Keywords Fibromyalgia - Nonpharmacologic therapy Pain management $\cdot$ Exercise $\cdot$ Aerobics $\cdot$ Strengthening . Aquatics · Flexibility $\cdot$ Pilates $\cdot$ Vibration $\cdot$ Tai chi $\cdot$ Nordic walking Physical activity. Randomized control trial . Review article - Cognitive behavioral therapy. Pain . Fatigue $\cdot$ Exercise progression $\cdot$ Exercise prescription . Adults $\cdot$ Yoga

\section{Introduction}

Fibromyalgia is a common chronic condition involving widespread pain, cognitive symptoms, nonrestorative sleep, fatigue, and a number of somatic symptoms [1], along with a reduced quality of life [2]. The prevalence of fibromyalgia in the United States is about $2 \%(95 \%$ CI, 1.4-2.7), or nearly 5 million people. Prevalence is higher among women $(3.4 \%)$ than men $(0.5 \%)$, rises in middle age, and peaks at $7.4 \%$ among those 70 to 79 years old [3]. Compared with age-matched control patients, women with

\author{
A. D. Danyliw \\ 218 Buckwold Cove, \\ Saskatoon, Saskatchewan S7N 4V9, Canada
}

T. J. Overend

School of Physical Therapy, University of Western Ontario,

1201 Western Road,

London, Ontario N6G 1H1, Canada

R. S. Richards

3355 Robinson Road,

North Vancouver, British Columbia V7J 3P7, Canada

A. Sawant

London Health Sciences Centre,

339 Windermere Road,

London, Ontario N6A 5A5, Canada 
fibromyalgia are less physically active as measured with accelerometers [4], have significantly lower perceived functional ability [5], and demonstrate impaired physical performance [5]. In contrast, a recent report found that about $2 \%$ of competitive-sport players have fibromyalgia [6], suggesting that persons with this condition can be very active. While regular exercise is one of the cornerstones of management of fibromyalgia, there still are many questions to resolve. For example, it is important to determine the most effective type of exercise (aerobic, strength, flexibility, or other); the intensity, duration, and frequency that should be prescribed; and the incidence and type of any related adverse effects. Interest in answering these questions is strong and the literature on this topic is growing steadily.

Exercise is defined as "planned, structured, and repetitive bodily movements that are performed to improve or maintain one or more components of physical fitness" [7]. Exercise is a subset of physical activity; the latter usually implies bodily movement with less structure and planning, but in the context of this review, refers to repetitive programs aimed at improving function and possibly improving some component of physical fitness. Several exercise studies over the past three decades demonstrated that persons with fibromyalgia are able to engage in moderate and even vigorous exercise; however, in many studies, participants experienced difficulties performing and adhering to vigorous and even moderate-intensity regimens because of increased fibromyalgia symptoms [8*0]. This paper reviews the effects of exercise and physical activity for individuals with fibromyalgia, summarizing recent reviews and describing new advances in research related to progressive exercise regimens (aerobic, strengthening, and flexibility interventions), more general lifestyle physical activity programs (i.e., self-selected, self-paced programs), and other forms of physical activity newly applied to fibromyalgia (e.g., tai chi, yoga, Pilates, and Nordic walking).

\section{Methods}

We examined all relevant reviews published from 2007 to 2011 to form a base for this paper. We then examined recent primary research studies retrieved using a keyword search (terms "exercise" and "fibromyalgia") of PubMed and Sport Discus between Jan 1, 2009 and Mar 1, 2011. Methodological quality of randomized controlled trials (RCTs) was evaluated by single reviewers using the Physiotherapy Evidence Database (PEDro; 10 items) [9]. Systematic reviews were rated for quality using the AMSTAR (assessment of multiple systematic reviews) scale (11 items) [10]. Scores are provided in Tables 1 and 2.

\section{Benefits of Exercise}

Since the first RCT examining the effects of exercise training for fibromyalgia was published in 1988 [11], growth in the body of literature has accelerated. In addition to growth in the number of RCTs, there is a trend toward increased sample size and improved methodological quality. Many forms of exercise training have been studied through high-quality RCTs, including aquatic and land-based exercise regimes involving aerobic, strength, flexibility, and mixed format exercise interventions. In addition to the growth in RCTs, there also has been growth in the number of review articles, with at least nine reviews published in the past 3 years.

Among several recent reviews summarizing the effects of exercise for fibromyalgia (see Table 1), three recent systematic reviews $[8 \cdot \bullet, 12,13 \cdot \bullet]$ have included metaanalyses of RCTs. In these reviews, aerobic exercise interventions were shown to reduce pain, fatigue, and depression and to improve health-related quality of life (HRQOL) and physical fitness [13••]. In Table 3, Cohen's rating system was used to summarize the magnitude of effect (effect size) for aerobic, strength, mixed, and aquatic exercise interventions. Strength training was associated with large improvements in global well-being and physical function, and mixed exercise training (combination of aerobic and/or strength and/or flexibility exercises) was shown to produce large improvements in pain and physical function $[8 \cdot \bullet$ ]. These reviews indicate that positive effects can be achieved with aquatic exercise $[8 \cdot \bullet, 13 \cdot \bullet]$, but Hauser et al. [13••] suggested that aquatic aerobic exercise may not be superior to land-based aerobic exercise. Kelley et al. [12] performed a meta-analysis on the effects of exercise (aerobic, strength, or combined aerobic and strength exercise) on global well-being in individuals with fibromyalgia and found a small, statistically significant effect in favor of exercise. When evaluating the benefits of exercise for people with fibromyalgia, it is important to consider possible adverse effects of exercise, such as an increase in symptoms (e.g., pain, stiffness, and fatigue) and musculoskeletal problems (e.g., plantar fasciitis, impingement syndrome). While adverse events have not been consistently reported, they are not uncommon [8・•] and may be associated with high drop-out rates in RCTs. As documented in a recent review [8••], the average drop-out rate among participants assigned to aerobic exercise groups $(22 \%)$ is higher $(P<0.05)$ than that of participants assigned to untreated control groups $(10 \%)$.

Clinically, exercise most often is delivered in conjunction with other treatments designed to manage symptoms in people with fibromyalgia, including medications, selfmanagement education programs, stress-management strategies, relaxation training, and electromyographic biofeed- 


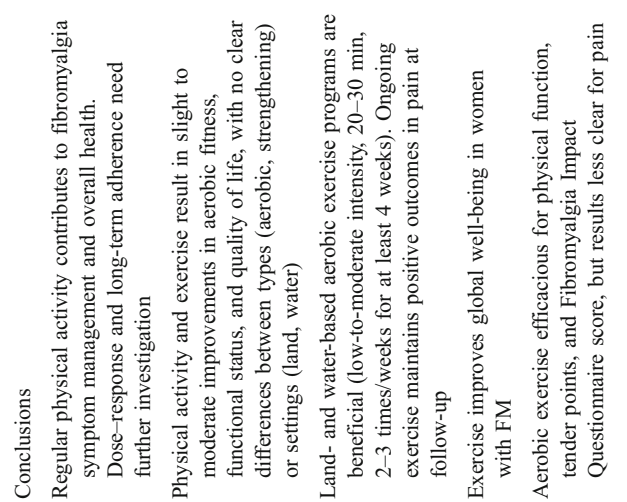

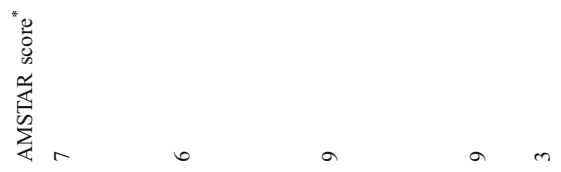

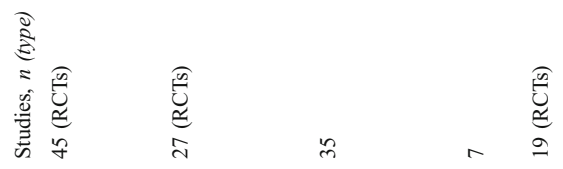

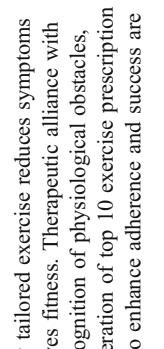

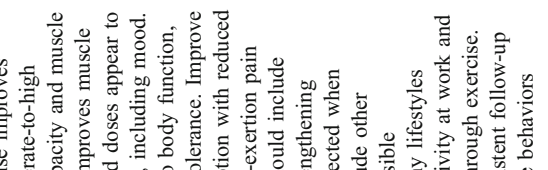

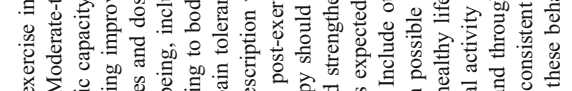

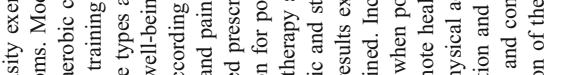

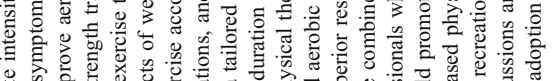
等

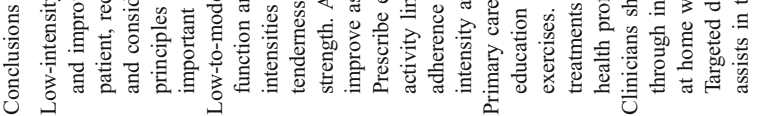

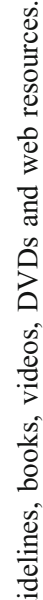

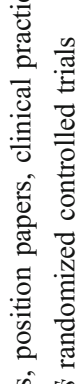

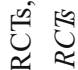

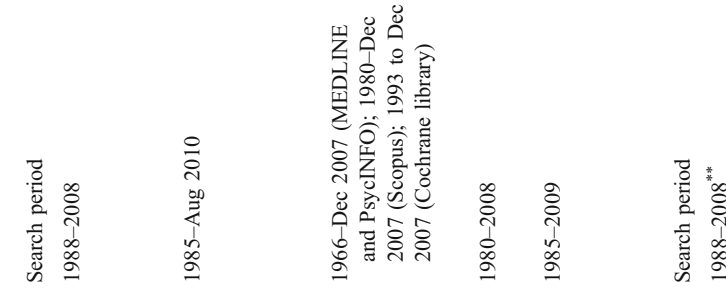

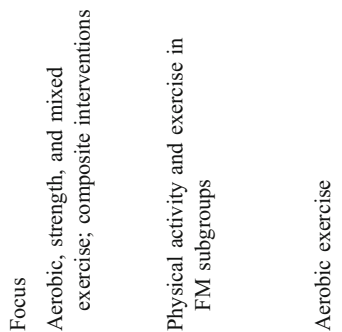

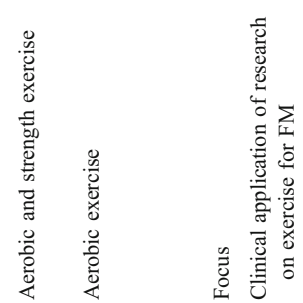<smiles></smiles><smiles>C1C2C3C1C23</smiles>

$\overleftrightarrow{z}$ $\mathbb{z} \quad \mathbb{z}$

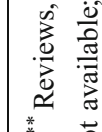
* (0) 言突 
Table 2 Fibromyalgia exercise intervention studies published between 2008 and 2011

\begin{tabular}{|c|c|c|c|}
\hline Study & PEDro score & Groups $(n)$ & Duration \\
\hline Altan et al. [25] & 7 & Pilates (25); control group (24) & 12 weeks; follow-up at 24 weeks \\
\hline Calandre et al. [20] & 5 & Stretching in warm pool (34); tai chi in warm pool (32) & 6 weeks; follow-up at 10 and 18 weeks \\
\hline Carson et al. [23] & 7 & Yoga (25); control group (28) & 8 weeks \\
\hline Etnier et al. [31] & 2 & $\begin{array}{l}\text { Mixed exercise (aerobic exercise, strength exercise, and } \\
\text { flexibility exercise; 8); control group (8) }\end{array}$ & 18 weeks \\
\hline Fontaine et al. [28] & 5 & Lifestyle physical activity (46); fibromyalgia education (38) & 12 weeks \\
\hline Fontaine et al. $[28,29]$ & 5 & Lifestyle physical activity (30); fibromyalgia education (23) & $\begin{array}{l}\text { 6-months and 12-months follow-up of } \\
\text { Fontaine [28] study from } 2010\end{array}$ \\
\hline Gusi et al. [26] & 9 & Whole-body vibration (21); control group (20) & 12 weeks \\
\hline Ide et al. [24] & 8 & Aquatic breathing exercise (20); control group (20) & 4 weeks \\
\hline Mannerkorpi et al. [16] & 8 & Aquatic exercise and education (81); education only (85) & 20 weeks \\
\hline Mannerkorpi et al. [27] & 7 & Supervised Nordic walking (34); low-intensity walking (33) & 15 weeks; follow-up at 6 months \\
\hline Sanudo et al. [57] & 7 & $\begin{array}{l}\text { Aerobic exercise (18); mixed exercise (aerobic, strength, } \\
\text { and flexibility exercises; } 21 \text { ); control group (21) }\end{array}$ & 24 weeks \\
\hline Sanudo et al. [19] & 7 & $\begin{array}{l}\text { Mixed exercise (aerobic exercise, strength exercise, and } \\
\text { flexibility exercises) and whole-body vibration (15); } \\
\text { exercise only (15) }\end{array}$ & 6 weeks \\
\hline Tomas-Carus et al. [17] & 9 & Exercise (17); control group (16) & 32 weeks \\
\hline Valencia et al. [58] & 6 & $\begin{array}{l}\text { Mixed (aerobic exercise, active range of motion exercises, } \\
\text { relaxation exercises; 8); abdo-diaphragmatic breathing and } \\
\text { myofascial stretching (10) }\end{array}$ & 12 weeks; follow-up at 36 weeks \\
\hline van Koulil et al. [35•] & 6 & $\begin{array}{l}\text { Pain persistence groups: Mixed exercise (aerobic exercise, } \\
\text { strength exercise, flexibility exercise, relaxation exercise, } \\
\text { and hydrotherapy), and CBT tailored to pain-persistence } \\
\text { model (39); wait-listed control group (45) } \\
\text { Pain avoidance groups: Mixed exercise (aerobic exercise, } \\
\text { strength exercise, flexibility exercise, relaxation exercise, } \\
\text { and hydrotherapy) and CBT tailored to pain-avoidance } \\
\text { model (29); wait-listed control group (45) }\end{array}$ & 10 weeks; follow-up at 6 months \\
\hline Wang et al. [22] & 8 & Yang-style tai chi (33); education and stretching (33) & 12 weeks; follow-up at 24 weeks \\
\hline
\end{tabular}

$C B T$ cognitive-behavioral therapy; PEDro Physiotherapy Evidence Database

back. Many such composite management programs have been investigated and shown to be effective $[8 \bullet \bullet$.
Results of primary intervention studies of exercise for people with fibromyalgia published in the past 3 years (see

Table 3 Effect sizes from meta-analyses based on Cohen's [56] categories*

\begin{tabular}{|c|c|c|c|c|}
\hline \multirow[t]{2}{*}{ Outcome } & \multicolumn{4}{|l|}{ Type of exercise } \\
\hline & Aerobic $^{\mathrm{a}}$ & Strength $^{\mathrm{b}}$ & Mixed $^{\mathrm{c}}$ & Aquatics \\
\hline Pain & $\begin{array}{l}\text { Small effect }[13 \cdot \bullet] \\
\text { no effect }[8 \cdot \bullet]\end{array}$ & $\begin{array}{l}\text { Large effect but limited } \\
\text { data }[8 \cdot \bullet]\end{array}$ & Large effect $[8 \bullet \bullet]$ & Medium effect $[8 \bullet \bullet, 13 \bullet \bullet]$ \\
\hline Fatigue & Small effect $[13 \bullet \bullet]$ & - & - & - \\
\hline Global health & Small effect $[8 \bullet \bullet, 13 \bullet \bullet]$ & $\begin{array}{l}\text { Large effect but limited } \\
\text { data }[8 \bullet \cdot]\end{array}$ & No effect $[8 \bullet \bullet]$ & Medium effect $[8 \bullet \bullet]$ \\
\hline $\begin{array}{l}\text { Physical function } \\
\text { (self-report, objectives) }\end{array}$ & Medium effect $[8 \bullet \bullet, 13 \bullet \bullet]$ & Large effect $[8 \bullet \bullet]$ & $\begin{array}{l}\text { Large cardiovascular effect, } \\
\text { medium strength effect }[8 \cdot \bullet]\end{array}$ & Large effect $[8 \bullet \bullet]$ \\
\hline Depression & $\begin{array}{l}\text { Small effect, medium } \\
\text { effect }[13 \bullet \cdot]\end{array}$ & $\begin{array}{l}\text { Large effect but limited } \\
\text { data }[8 \cdot \bullet]\end{array}$ & No effect $[8 \bullet \bullet$ & $\begin{array}{l}\text { Large effect but limited } \\
\text { data }[8 \cdot \bullet]\end{array}$ \\
\hline
\end{tabular}

*Cohen's [56] categories to evaluate the magnitude of effect sizes (small effect: $0.2-0.49$; medium effect: $0.5-0.79$; large effect: $\geq 0.8$ ).

${ }^{a}$ Walking, games, aerobic dance, treadmill, cycling;

${ }^{\mathrm{b}}$ Progressive resistance exercise;

${ }^{\mathrm{c}}$ Two or more of strength, aerobic, flexibility) 
Table 2) are consistent with the earlier studies described in the most current meta-analyses: 1 ) aerobic training improves peak oxygen uptake and decreases pain intensity and fatigue [14]; 2) strength training reduces severity of fibromyalgia symptoms [15]; 3) aquatic exercise is associated with improvements in pain [16, 17], HRQOL [16], physical function [16], muscle strength [17], emotional and mental health [17], and vitality [17]; and 4) multidisciplinary programs (pool and land-based exercise and psychoeducational sessions) improve tenderness severity [18] and lower body flexibility, but not physical fitness [19]. Consistent with the few studies that have examined the effects of flexibility exercise [8••], one recent study by Calandre et al. [20] comparing the effects of pool-based stretching and ai chi (water-based tai chi) found improvements in symptoms in the ai chi group; however, there were no significant betweengroup differences.

A recent study using medical imaging provided confirmation that increased physical activity has a positive effect on pain perception in women with fibromyalgia. McLoughlin et al. [21] demonstrated that higher levels of self-reported and accelerometer-monitored physical activity in women with fibromyalgia were associated with decreases in pain ratings and "greater responses in pain regulatory brain regions while receiving painful stimuli."

\section{Exercise Interventions Newly Applied in Fibromyalgia}

Recently, researchers have begun to explore the effects of a wide range of exercise techniques that extend beyond more conventional exercise training modes (e.g., low-impact aerobic training and strength training). Some of the interventions being explored are modeled on longhonored holistic approaches used in China and other Asian countries (e.g., tai chi, chi gong, and yoga) or Nordic countries (e.g., Nordic walking); some arise from technologies newly applied to exercise (e.g., vibration); and others represent rethinking the prescriptive approach to physical activity (e.g., lifestyle physical activity).

\section{Tai Chi, Yoga, Breathing Exercises, and Pilates}

Mind-body forms of exercise (e.g., tai chi, yoga, and Pilates) are considered holistic, encompassing physical, psychosocial, emotional, spiritual, and behavioral elements, which may be especially beneficial for those with fibromyalgia. Wang et al. [22] recently reported the results of an RCT of twice weekly tai chi sessions, delivered for 12 weeks, for men and women with fibromyalgia $(n=66)$. In this study, tai chi sessions included a warm-up and selfmassage, a review of tai chi principles, and practice with tai chi movements, breathing techniques, and relaxation.
Participants demonstrated large improvements in HRQOL (as measured by the Fibromyalgia Impact Questionnaire [FIQ] and Short Form-36 health survey questionnaire [SF-36]) beyond what typically have been reported for exercise and pharmacologic interventions.

The effects of a yoga program including traditional yoga poses, meditation and breathing exercises, yoga-based coping presentations, and group discussions recently were reported [23]. In the yoga group, 22 women attended weekly supervised 120-min yoga sessions and were encouraged to practice yoga at home five to seven times per week for 8 weeks. On retesting, the intervention participants showed improvements in standardized measures of a wide range of fibromyalgia symptoms and functional deficits. Ide et al. [24] utilized a yoga breathing technique (pranayama) in conjunction with range of motion and relaxation exercises in water for women with fibromyalgia (1-h sessions, four times per week for 4 weeks). Efficacy was assessed through self-report questionnaires completed by 18 women in the intervention group and 17 women in the control group who received no exercise intervention. Significant improvements were demonstrated on a number of SF-36 and FIQ components, as well as global pain rating and dyspnea scores.

Pilates recently has become a fast-growing popular form of exercise recommended for healthy individuals and for those engaged in rehabilitation. Pilates exercises emphasize core strengthening, posture, and coordination of breathing with movement, combining Asian and Western techniques. In 2009, Altan et al. [25] investigated the effects of Pilates training in 49 women with fibromyalgia (ages 24-63 year). They demonstrated improvements in pain visual analogue scale scores and FIQ results compared to control participants who performed home-based relaxation and stretching exercises. However, after an additional 12 weeks of follow-up, there were no differences between the groups.

Taken together, these studies highlight that a more mindbody integrative approach may be beneficial for people with fibromyalgia. Study results are promising, and further research comparing mind-body techniques to more conventional exercise approaches are needed.

\section{Vibration and Nordic Walking}

Whole-body vibration represents a relatively new exercise alternative for people with fibromyalgia. Gusi et al. [26] reported that after 12 weeks, women with fibromyalgia who performed squats while standing on a vibration platform (six partial squats held 45-60 s, three times per week; vibration parameters: $12.5 \mathrm{~Hz}$, vertical amplitude $3 \mathrm{~mm}$ ) demonstrated improvements in dynamic balance, as assessed on the Biodex Balance System (Biodex Medical 
Systems, inc., Shirley, NY), that were not seen in control patients who maintained usual daily activities. Another group of researchers also recently investigated the effects of vibration, comparing results in 30 postmenopausal women with fibromyalgia randomly assigned to a combined exercise/vibration program (aerobic, strengthening, and flexibility exercises twice weekly, plus training consisting of unilateral static squats performed on a vibration platform three times weekly) or a traditional exercise program (aerobic, strengthening, and flexibility exercises twice weekly) [19]. Both exercise groups demonstrated improvements in FIQ scores and lower extremity maximal power output; however, the group that completed vibration training also demonstrated improvements in the SF-36 global score, suggesting whole-body vibration may impart additional benefits when combined with traditional mixed exercise programs.

Mannerkorpi et al. [27] investigated tolerance to moderate to high-intensity outdoor Nordic walking (walking with poles) in women with fibromyalgia to determine whether functional capacity and pain improved. They found that this form of walking, which places increased demands on the upper body, was generally well tolerated among those who completed the 45-min training sessions twice weekly for 15 weeks ( 29 of 34 women). Nordic walkers demonstrated significant improvements in the 6-min walk test and FIQ physical scores, as well as reduced heart rates at similar workloads during submaximal cycle ergometer tests compared to women in the control group who exhibited no changes with a low-intensity outdoor walking program.

\section{Lifestyle Physical Activity}

Recent work by McLoughlin et al. [4] indicates that most women with fibromyalgia are less physically active than age-matched healthy women. Although exercise is recommended, symptoms often make it difficult for individuals with fibromyalgia to exercise consistently enough to derive benefits. To address this deficit, Fontaine et al. [28] attempted to improve exercise adherence by encouraging increased home-based daily physical activity versus attendance at fibromyalgia education sessions. In this 12-week trial, individuals randomly assigned to the lifestyle physical activity group increased their average daily pedometer step count by $54 \%$ and reported improved fibromyalgia symptoms (FIQ total score) and lower pain levels compared to the education group. Participants who completed $73 \%$ of the sessions in the 12-week trial then were followed 6 and 12 months after the intervention [29]. At both follow-up assessments, changes in physical activity (steps per day), function, and pain were not maintained participant in the lifestyle physical activity group; however, participants reported greater perceived improvement.
While it is encouraging to see that programs that promote increased physical activity in daily life can produce clinically relevant changes, additional research should focus on supports to maintain increased activity levels and/or the benefits of increasing lifestyle physical activity for improving tolerance in subsequent formal exercise programs.

\section{Focus on Cognitive Function}

A broad spectrum of outcome measures has been used in research to explore the effects of exercise for fibromyalgia $[8 \cdot \bullet]$. Outcomes have been measured for such variables as pain, tenderness, sleep, mental and physical health, physical fitness, and quality of life. Recently, Wolfe et al. [1] drew attention to the importance of cognitive symptoms in fibromyalgia. These cognitive symptoms, which are sometimes collectively referred to as fibrofog [30], often are described as among the most debilitating symptoms of the condition. However, researchers only recently have examined the effects of physical activity or exercise on fibrofog, mental health, emotional health, and other cognitive performance outcomes. Etnier et al. [31] reported no significant improvements in cognitive ability, memory, attention, and executive function in women with fibromyalgia who completed 1-h exercise sessions (walking, light resistance exercises, static bridging, and stretching) three times per week. While it should be noted that the study was underpowered $(n=16)$, effect sizes suggest positive effects of exercise on 5 of 7 cognitive performance measures and indicate that further research in this area may be warranted.

\section{Factors Affecting Response to Exercise}

The population of individuals with fibromyalgia is diverse with respect to symptoms (variety, severity, and duration), physical fitness levels, psychological characteristics, and preferences for type of exercise and physical activity [16, 32-34], [35•, 36•]. Recent research has shown that many of the key features of the condition are interrelated. For example, greater levels of fibromyalgia symptoms are associated with lower levels of aerobic fitness [34], and newly diagnosed individuals have higher levels of psychological distress regarding their fibromyalgia [35•]. Researchers have suggested that exercise effectiveness may depend upon these specific characteristics [16, 32-34, 37] and recommend customizing exercise programs based on variables other than or related to fitness characteristics $[16,32-34,35 \bullet, 36 \bullet]$. For 
example, in a recent RCT, the intervention (exercise and cognitive behavioral therapy) was individually tailored according to psychological risk factors (pain-persistence and pain-avoidance patterns) [35•]. Significant improvements in pain, function, mood, anxiety, and HRQOL were observed in the intervention group.

\section{Advice for Clinicians and Consumers}

The goals of physical activity and exercise training are to improve physical fitness and function, reduce fibromyalgia symptoms, and optimize overall health and well-being. Because sedentary lifestyles [4] and the general deconditioning associated with fibromyalgia may place this population at increased risk for a number of chronic diseases $[8 \cdot \bullet$, optimizing overall health and well-being is particularly important. Furthermore, improved physical fitness permits daily activities to be performed at a lower percentage of maximum capacity and possibly more efficiently, with less likelihood of increasing symptoms [38••]. Additional goals depend on baseline body functions and symptom severity along with individual preferences and motivations [16, 33, 39]. Recent RCTs and literature reviews provide considerable insight into physical activity and exercise programs to help individuals with fibromyalgia achieve these goals.

To gain optimal benefits and ensure long-term adherence, care must be taken to avert exercise-related pain and fatigue and musculoskeletal injury. A recent systematic review suggests that warm water-based exercise may be superior in terms of adherence [8・•], while approaches such as tai chi [22] and lifestyle physical activities that include walking at self-selected intensity [28, 29] appear well-tolerated.

It seems there may be a narrow therapeutic window in fibromyalgia, with too little exercise failing to yield benefits and too much exercise exacerbating symptoms [38••]. While avoiding excessive workloads is important in central sensitization disorders such as fibromyalgia [8••, 33, 39, 40], people with fibromyalgia can exercise at workloads necessary to achieve training effects and gain long-term benefits (i.e., as recommended by the American College of Sports Medicine [41]). For example, a recent meta-analysis indicated that low to moderate-intensity, but not lowintensity, aerobic exercise reduced pain [13••], and 29 of 34 women randomly assigned to Nordic walking in a recent RCT tolerated moderate to high-intensity aerobic exercise [27]. Several studies also have demonstrated that participants with fibromyalgia showed similar training effects as healthy control patients, with improvements in fatigue, depression, and pain in response to progressive strengthtraining exercise [42-44].
Prescribing for Success: Lessons from Current Evidence

Successful exercise prescription for this clinical population requires finesse $[38 \cdot \bullet$. The process begins with a thorough medical evaluation including cardiovascular health screening [45] and management of symptoms. Common comorbidities, sleep disturbances, autonomic dysfunction, and

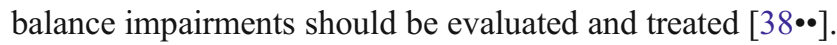
Individual characteristics, such as physical fitness, function, and symptom severity, along with goals and personal preferences for type and setting of physical activity should be accounted for when developing programs $[32,33,38 \bullet \cdot]$.

\section{Exercise Type}

While most consistent results have been demonstrated for aerobic and strengthening exercise [8$\bullet \bullet]$, there is no optimal application overall [33]. With this in mind, including different types of exercise in the same session or in different sessions is recommended [33]. While there is only limited evidence supporting flexibility exercise in the management of fibromyalgia $[8 \cdot \bullet, 20]$, it is common practice to include stretching as part of the exercise cooldown session [8••]. With these considerations in mind, patient preferences and available settings should guide exercise prescription. Nordic walking programs and mindbody approaches, including tai chi or yoga, also hold considerable promise and appear to be viable options.

While a recent meta-analysis indicated that aquatic aerobic exercise does not produce superior results compared to similarly intense land exercise [13••], other reviews (that did not employ meta-analysis) suggest slight additional benefits in reducing pain and depression with water strengthening and aerobic exercise [33] and in sleep quality and mood improvement for aquatic aerobic exercise [40]. Exercising in water may be particularly valuable for severely deconditioned individuals or for those with particularly high levels of pain or distress $[8 \bullet \bullet$.

\section{Exercise Intensity}

Most authors recommend a gradual progression from lowintensity exercise [38••, 40], using the "start low and go slow" approach [38・•] with the goal of achieving at least moderate intensity $[8 \cdot \bullet, 38 \bullet \bullet, 46]$. Strengthening programs should begin with lower resistance levels than agepredicted norms $[38 \cdot \bullet]$. The intensity and duration of exercise sessions should be reduced when significant postexertion pain or fatigue is experienced $[32,33,39,40]$, and the intensity increased by $10 \%$ after 2 weeks of exercise without exacerbating symptoms $[38 \bullet \bullet]$.

In a recent report [46], researchers used mathematical modeling to determine how age-predicted heart rate 
formula can be used for people with fibromyalgia. They also calculated target heart rate zones for aerobic training within the anaerobic threshold, a training intensity range shown to improve cardiorespiratory fitness. The researchers found that maximum heart rate could be adequately predicted using either $(208-[0.7 \times$ age $])$ or $(220-$ age $)$ and recommended that for sedentary individuals with fibromyalgia, training within the anaerobic threshold could be achieved at $52 \%$ to $60 \%$ of the heart rate reserve or at $75 \%$ to $85 \%$ of predicted maximum heart rate.

\section{Delivery Options}

Supervised programs encouraging participants to perform short bouts of self-selected physical activity show promise as a first step in building exercise programs for sedentary individuals. Use of exercise diaries and pedometers during exercise and physical activity (techniques found effective for increasing physical activity levels in healthy populations) may help to motivate individuals with fibromyalgia [47].

While we recommend a multidisciplinary approach for management of fibromyalgia, we also advocate exercise and physical activity prescription in conjunction with education by health care providers such as physical therapists who are in primary or monodisciplinary settings [39]. Self-management and coping strategies also may be enhanced by participation in group education programs, as recommended by several clinical practice guidelines for fibromyalgia [39]. This approach is consistent with models of care for management of chronic disease [48, 49].

\section{Promoting Adherence by Facilitating Self-efficacy for Exercise}

Promoting self-efficacy is recommended as one of the 10 top exercise prescription principles for individuals with fibromyalgia $[38 \bullet \bullet$, contributing to exercise performance and subsequent adherence to exercise and physical activity regimens. Self-efficacy involves "having, or gaining, the confidence that one can complete a task, such as regular participation in exercise (efficacy expectation)" and “... believing that completing a task results in the desired effect, such as fitness or symptom control (efficacy outcome)" $[38 \bullet \cdot$. In essence, individuals must believe that they can successfully perform an activity before they even consider undertaking it.

Jones and Liptan [38••] drew on Bandura's [50, 51] extensive work on self-efficacy. They cite Bandura [50] in describing four ways for clinicians to help individuals with fibromyalgia gain self-efficacy, including mastery, symptom reduction, modeling, and verbal persuasion. Mastery includes designing a program that is realistic and achiev- able, helping patients to meet their goals by "starting low, going slow" and celebrating success. Avoiding excessive workloads that lead to symptom flares and improving symptoms over the long term contributes to the second element, symptom reduction. Modeling is demonstrated when group participants who successfully perform exercise programs set a positive example for others to follow, while verbal persuasion includes assuring patients that they can achieve their physical activity and exercise goals. The latter approach can be enhanced via a trusting therapeutic relationship [38••, 52] and motivational interviewing techniques $[8 \cdot \bullet]$.

\section{Future Directions for Research}

Priorities for research on exercise for individuals with fibromyalgia were clearly laid out by Brachaniec et al. [53] using the EPICOT (evidence, population, intervention, comparison, outcomes, and time stamp) framework recommended by editors of the British Medical Journal [54]. Further research examining the effects of exercise and physical activity for people with fibromyalgia is needed 1) to elucidate the dose-response curve for exercise intensity, frequency, and duration on symptoms; 2) to evaluate the long-term effects on health in this population; 3) to determine how long positive effects are sustained; and 4) to determine synergistic effects of exercise and other management strategies. Continued and extensive investigation of the heterogeneity of the fibromyalgia population with regards to the short- and long-term response patterns of subpopulations to exercise/physical activity prescription may lead to new types of program customization to improve exercise adherence and optimize the benefits of exercise and physical activity.

\section{Conclusions}

The benefits of exercise training for individuals with fibromyalgia are well documented in recent high-quality reviews and meta-analyses and include reduction of pain and depression and improvement in global health and physical function. RCTs examining the effects of exercise on symptoms, function, fitness, and quality of life in fibromyalgia have grown in number and quality. Exercise options that have been investigated have recently expanded to include tai chi, chi gong and yoga, Nordic walking, vibration, and lifestyle physical activity. Research shows that persons with fibromyalgia are able to engage in moderate and even vigorous exercise; however, participants have experienced difficulties performing and adhering to vigorous and even moderate-intensity 
regimes because of increased fibromyalgia symptoms. Undoubtedly, successful exercise prescription requires finesse. To gain optimal benefits and ensure long-term adherence, care must be taken to avert exercise-related pain and fatigue and musculoskeletal injury. Individual characteristics such as physical fitness, function and symptom severity, goals and personal preferences should be taken in consideration when developing programs. A gradual intensity progression for deconditioned individuals with fibromyalgia toward "moderate" intensity is recommended. Although multidisciplinary management is advocated, clinicians in primary care roles ought to ensure that their clients with fibromyalgia are initiated and supported in adopting active lifestyles that include regular exercise. The fundamental questions raised in the introduction remain only partially answered, but if research on exercise for fibromyalgia continues to accumulate at the current pace, clinicians will have evidence needed to aid in designing and recommending optimal exercise programs for this population.

Acknowledgements The authors would like to acknowledge the contributions of Louise Falzon who assisted with the literature search and Joelle Harris who helped with the completion of this manuscript.

Disclosures No potential conflicts of interest relevant to this article were reported.

Open Access This article is distributed under the terms of the Creative Commons Attribution Noncommercial License which permits any noncommercial use, distribution, and reproduction in any medium, provided the original author(s) and source are credited.

\section{References}

Papers of particular interest, published recently, have been highlighted as:

- Of importance

•. Of major importance

1. Wolfe F, Clauw DJ, Fitzcharles MA, et al. The American College of Rheumatology preliminary diagnostic criteria for fibromyalgia and measurement of symptom severity. Arthritis Care Res. 2010;62:600-10

2. Mease PJ, Arnold LM, Crofford LJ, et al. Identifying the clinical domains of fibromyalgia: contributions from clinician and patient delphi exercises. Arthritis Rheum. 2008;59:952-60.

3. Lawrence RC, Felson DT, Helmick CG, et al. Estimates of the prevalence of arthritis and other rheumatic conditions in the United States. Part II. Arthritis Rheum. 2008;58:26-35.

4. McLoughlin MJ, Colbert LH, Stegner AJ, Cook DB. Are women with fibromyalgia less physically active than healthy women? Med Sci Sports Exerc. 2011;43:905-12.

5. Jones CJ, Rutledge DN, Aquino J. Predictors of physical performance and functional ability in people $50+$ with and without fibromyalgia. J Aging Phys Act. 2010;18:353-68.
6. Inanici $\mathrm{F}$, Ozdemir $\mathrm{O}$, Aydog $\mathrm{T}$, et al. The frequency of fibromyalgia in sport professionals. Rheumatol Int. 2010; http:// www.springerlink.com/content/5360rt1762170546/fulltext.pdf.

7. Howley ET. Type of activity: resistance, aerobic and leisure versus occupational physical activity. Med Sci Sports Exerc. 2001;33: S364-9.

8. •• Busch AJ, Overend TJ, Schachter CL: Fibromyalgia treatment: the role of exercise and physical activity. Int J Clin Rheumtol. 2009;4:343-80. This comprehensive systematic review and metaanalysis of RCTs up to 2008 examines the effects of all forms of exercise interventions, including aerobic, strength, flexibility, mixed aerobic and strength, and combination interventions (exercise when combined with nonexercise) for individuals with fibromyalgia.

9. Maher CG, Sherrington C, Herbert RD, et al. Reliability of the PEDro scale for rating quality of randomized controlled trials. Phys Ther. 2003;83:713-21.

10. Shea BJ, Grimshaw JM, Wells GA, et al. Development of AMSTAR: a measurement tool to assess the methodological quality of systematic reviews. BMC Med Res Methodol. 2007;7:10. http://www.biomedcentral.com/1471-2288/7/10.

11. McCain GA, Bell DA, Mai FM, Halliday PD. A controlled study of the effects of a supervised cardiovascular fitness training program on the manifestations of primary fibromyalgia. Arthritis Rheum. 1988;31:1135-41.

12. Kelley GA, Kelley KS, Hootman JM, Jones DL. Exercise and global well-being in community-dwelling adults with fibromyalgia: a systematic review with meta-analysis. BMC Public Health. 2010;10:198. http://www.biomedcentral.com/1471-2458/10/198.

13. • Hauser W, Klose P, Langhorst J, et al. Efficacy of different types of aerobic exercise in fibromyalgia syndrome: a systematic review and meta-analysis of randomised controlled trials. Arthritis Res Ther. 2010;12:R79. http://arthritis-research.com/content/12/3/ $\mathrm{R} 79$. In this systematic review and meta-analysis of aerobic exercise interventions for persons with fibromyalgia, subgroup analyses and head-to-head comparisons were used to assess which types, volumes, and intensities of aerobic exercise are most effective.

14. Dinler M, Diracoglu D, Kasikcioglu E, et al. Effect of aerobic exercise training on oxygen uptake and kinetics in patients with fibromyalgia. Rheumatol Int. 2009;30:281-4.

15. Kingsley JD, McMillan V, Figueroa A. The effects of 12 weeks of resistance exercise training on disease severity and autonomic modulation at rest and after acute leg resistance exercise in women with fibromyalgia. Arch Phys Med Rehabil. 2010;91:1551-7.

16. Mannerkorpi K, Nordeman L, Ericsson A, Arndorw M. Pool exercise for patients with fibromyalgia or chronic widespread pain: a randomized controlled trial and subgroup analyses. J Rehabil Med. 2009;41:751-60.

17. Tomas-Carus P, Gusi N, Hakkinen A, et al. Improvements of muscle strength predicted benefits in HRQOL and postural balance in women with fibromyalgia: an 8-month randomized controlled trial. Rheumatology (Oxford). 2009;48:1147-51.

18. Carbonell-Baeza A, Aparicio VA, Ortega FB, et al. Does a 3-month multidisciplinary intervention improve pain, body composition and physical fitness in women with fibromyalgia? $\mathrm{Br} \mathrm{J}$ Sports Med. 2010; http://bjsm.bmj.com/content/early/2010/06/01/ bjsm.2009.070896. full.pdf?sid=8ec58377-62bf-4900-a5183338 af5balda.

19. Sanudo B, de Hoyo M, Carrasco L, et al. The effect of 6-week exercise programme and whole body vibration on strength and quality of life in women with fibromyalgia: a randomised study. Clin Exp Rheumatol. 2010;28:S40-5.

20. Calandre EP, Rodriguez-Claro ML, Rico-Villademoros F, et al. Effects of pool-based exercise in fibromyalgia symptomatology 
and sleep quality: a prospective randomised comparison between stretching and Ai Chi. Clin Exp Rheumatol. 2009;27:S13-20.

21. McLoughlin MJ, Stegner AJ, Cook DB. The relationship between physical activity and brain responses to pain in fibromyalgia. $\mathrm{J}$ Pain 2011;1-12. http://www.sciencedirect.com/.

22. Wang $\mathrm{C}$, Schmid $\mathrm{CH}$, Rones R, et al. A randomized trial of tai chi for fibromyalgia. N Engl J Med. 2010;363:743-54.

23. Carson JW, Carson KM, Jones KD, et al. A pilot randomized controlled trial of the Yoga of awareness program in the management of fibromyalgia. Pain. 2010;151:530-9.

24. Ide MR, Laurindo LMM, Rodrigues-Junior AL, Tanaka C. Effect of aquatic respiratory exercise-based program in patients with fibromyalgia. Int J Rheum Dis. 2008;11:131-40.

25. Altan L, Korkmaz N, Bingol U, Gunay B. Effect of Pilates training on people with fibromyalgia syndrome: a pilot study. Arch Phys Med Rehabil. 2009;90:1983-8.

26. Gusi N, Parraca JA, Olivares PR, et al. Tilt vibratory exercise and the dynamic balance in fibromyalgia: a randomized controlled trial. Arthritis Care Res. 2010;62:1072-8.

27. Mannerkorpi K, Nordeman L, Cider A, Jonsson G. Does moderate-to-high intensity Nordic walking improve functional capacity and pain in fibromyalgia? A prospective randomized controlled trial. Arthritis Res Ther. 2010;12:R189. http://arthritisresearch.com/content/12/5/R189.

28. Fontaine KR, Conn L, Clauw DJ. Effects of lifestyle physical activity on perceived symptoms and physical function in adults with fibromyalgia: results of a randomized trial. Arthritis Res Ther. 2010;12:R55. http://arthritis-research.com/content/12/2/R55.

29. Fontaine KR, Conn L, Clauw DJ. Effects of lifestyle physical activity in adults with fibromyalgia: results at follow-up. J Clin Rheumatol. 2011;17:64-8.

30. Leavitt F, Katz RS, Mills M, Heard AR. Cognitive and dissociative manifestations in fibromyalgia. J Clin Rheumatol. 2002;8:77-84.

31. Etnier JL, Karper WB, Gapin JI, et al. Exercise, fibromyalgia, and fibrofog: a pilot study. J Phys Act Health. 2009;6:239-46.

32. Mannerkorpi K. Physical activity and body functions in patients with fibromyalgia syndrome. J Musculoskelet Pain. 2009;17:28794.

33. Cazzola M, Atzeni F, Salaffi F, et al. Which kind of exercise is best in fibromyalgia therapeutic programmes? A practical review. Clin Exp Rheumatol. 2010;28:S117-24.

34. Sanudo B, Galiano D. Using cardiovascular parameters and symptom severity to prescribe physical activity in women with fibromyalgia. Clin Exp Rheumatol. 2009;27:S62-6.

35. • van Koulil S, van Lankveld W, Kraaimaat FW, et al.: Tailored cognitive-behavioral therapy and exercise training for high-risk patients with fibromyalgia. Arthritis Care Res. 2010;62:1377-85. The authors report the results of an RCTexamining customization of exercise interventions for women with fibromyalgia based on coping style (pain avoidance vs. pain persistence).

36. - Newcomb LW, Koltyn KF, Morgan WP, Cook DB. The influence of preferred versus prescribed exercise on pain in fibromyalgia. Med Sci Sports Exerc. 2010; Epub ahead of print. The authors reported the results of an $R C$ Texamining customization of exercise interventions for women with fibromyalgia based on participant preference.

37. Hoeger Bement MK, Weyer A, Hartley S, et al. Pain perception after isometric exercise in women with fibromyalgia. Arch Phys Med Rehabil. 2011;92:89-95.

38. • Jones KD, Liptan GL. Exercise interventions in fibromyalgia: clinical applications from the evidence. Rheum Dis Clin North Am. 2009;35:373-91. This topical review of exercise interventions for fibromyalgia presents physiological obstacles and 10 principles for exercise prescription.

39. Nijs J, Mannerkorpi K, Descheemaeker F, Van Houdenhove B. Primary care physical therapy in people with fibromyalgia: opportunities and boundaries within a monodisciplinary setting. Phys Ther. 2010;90:1815-22.

40. Thomas EN, Blotman F. Aerobic exercise in fibromyalgia: a practical review. Rheumatol Int. 2010;30:1143-50.

41. Busch AJ, Barber KA, Overend TJ, et al. Exercise for treating fibromyalgia syndrome. Cochrane Database Syst Rev. 2007; CD003786. doi: 10.1002/14651858.CD003786.pub2.

42. Hakkinen A, Hakkinen K, Hannonen P, Alen M. Strength training induced adaptations in neuromuscular function of premenopausal women with fibromyalgia: comparison with healthy women. Ann Rheum Dis. 2001;60:21-6.

43. Hakkinen K, Pakarinen A, Hannonen P, et al. Effects of strength training on muscle strength, cross-sectional area, maximal electromyographic activity, and serum hormones in premenopausal women with fibromyalgia. J Rheumatol. 2002;29:1287-95.

44. Valkeinen H, Hakkinen K, Pakarinen A, et al. Muscle hypertrophy, strength development, and serum hormones during strength training in elderly women with fibromyalgia. Scan J Rheumatol. 2005;34:309-14.

45. American College of Sports Medicine. ACSM's guidelines for exercise testing and prescription. Philadelphia: Lippincott Williams \& Wilkins; 2009.

46. Lemos MC, Valim V, Zandonade E, Natour J. Intensity level for exercise training in fibromyalgia by using mathematical models. BMC Musculoskelet Disord. 2010;11:54. http://www.biomedcentral. com/1471-2474/11/54.

47. Tudor-Locke C, Lutes L. Why do pedometers work?: a reflection upon the factors related to successfully increasing physical activity. Sports Med. 2009;39:981-93.

48. Lorig KR, Sobel DS, Ritter PL, et al. Effect of a self-management program on patients with chronic disease. Eff Clin Pract. 2001;4:256-62.

49. Wagner EH, Austin BT, Davis C, et al. Improving chronic illness care: translating evidence into action. Health Aff (Millwood). 2001;20:64-78.

50. Bandura A. Self-efficacy: toward a unifying theory of behavioral change. Psychol Rev. 1977;84:191-215.

51. Bandura A. Social foundation of thought and action. Englewood Cliffs: Prentice Hall; 1986.

52. Busch AJ, Thille P, Barber KA, et al. Best practice: E-Modelprescribing physical activity and exercise for individuals with fibromyalgia. Physiother Theory Pract. 2008;24:151-66.

53. Brachaniec M, DePaul V, Elliott M, et al. Partnership in action: an innovative knowledge translation approach to improve outcomes for persons with fibromyalgia (Editorial). Physiother Canada. 2009;61:123-7.

54. Brown P, Brunnhuber K, Chalkidou $\mathrm{K}$, et al. How to formulate research recommendations. BMJ. 2006;333:804-6.

55. Rooks DS. Talking to patients with fibromyalgia about physical activity and exercise. Curr Opin Rheumatol. 2008;20:208-12.

56. Cohen J. Statistical power analysis for the behavioral sciences. Hillsdale: Lawrence Erlbaum Associates; 1988.

57. Sanudo B, Galiano D, Carrasco L, et al. Aerobic exercise versus combined exercise therapy in women with fibromyalgia syndrome: a randomized controlled trial. Arch Phys Med Rehabil. 2010;91:183843.

58. Valencia M, Alonso B, Alvarez MJ, et al. Effects of 2 physiotherapy programs on pain perception, muscular flexibility, and illness impact in women with fibromyalgia: a pilot study. J Manipulative Physiol Ther. 2009;32:84-92. 